\title{
A low-fat diet enriched in fish oil increased lipogenesis and fetal outcome of C57BL/6 mice
}

\author{
Olatunji A Akerele and Sukhinder K Cheema \\ Department of Biochemistry, Memorial University of Newfoundland, St John's, Newfoundland, Canada \\ Correspondence should be addressed to S K Cheema; Email: skaur@mun.ca
}

Reproduction (2021) $\mathbf{1 6 1}$ X1

The authors apologise for some accidental errors in their article published in this journal (vol 154 iss 2; pages 153-165). The corrections are listed below

1. The authors regret a scale conversion error in Figure 3A. The correct figure is published below with the accurate scale conversion on the Y-axis.

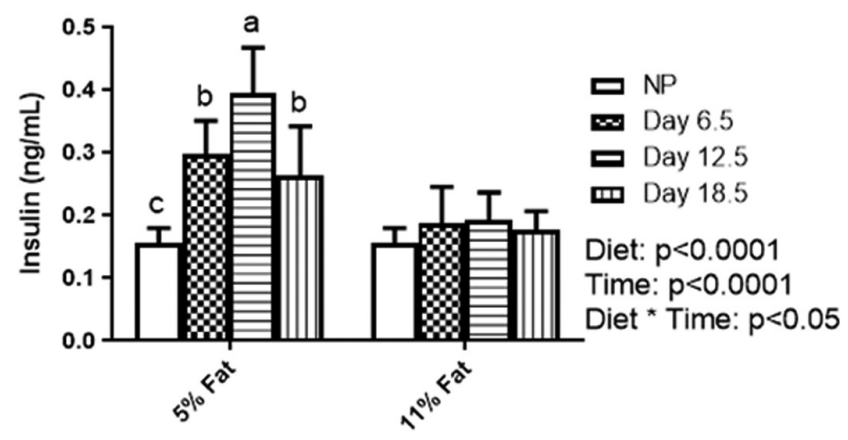

2. The authors report a typographical error in the method section, under the section titled 'Cholesterol efflux assay', line 3 where the correct cell density is $2 \times 10^{5}$ cells/well and not as published.

3. The authors also regret decimal point errors in Table 5. The correct table with accurate decimal points is published below

Table 5 Pregnancy outcomes.

\begin{tabular}{|c|c|c|c|c|c|c|c|c|c|}
\hline Pregnancy & & $5 \%$ Fat & & & $11 \%$ Fat & & & Main effec & \\
\hline Outcomes & Day 6.5 & Day 12.5 & Day 18.5 & Day 6.5 & Day 12.5 & Day 18.5 & Diet & Time & Diet $\times$ Time \\
\hline $\begin{array}{l}\text { Fetal weight } \\
\text { (g) }\end{array}$ & $\mathrm{N} / \mathrm{A}$ & $0.90 \pm 0.02^{b}$ & $1.11 \pm 0.12^{\mathrm{a}}$ & N/A & $0.90 \pm 0.01^{b}$ & $1.08 \pm 0.12^{\mathrm{a}}$ & NS & $P<0.0001$ & NS \\
\hline $\begin{array}{l}\text { Placental } \\
\text { weight (g) }\end{array}$ & N/A & $0.059 \pm 0.14^{b}$ & $0.086 \pm 0.15^{a}$ & $\mathrm{~N} / \mathrm{A}$ & $0.054 \pm 0.14^{b}$ & $0.070 \pm 0.20^{\mathrm{a}}$ & NS & $P<0.05$ & NS \\
\hline $\begin{array}{l}\text { Whole uterine } \\
\text { weight }(\mathrm{g})\end{array}$ & $0.29 \pm 0.07^{d}$ & $2.85 \pm 0.60^{c}$ & $13.48 \pm 3.14^{\mathrm{a}}$ & $0.32 \pm 0.05^{d}$ & $2.89 \pm .36^{c}$ & $9.45 \pm 4.53^{b}$ & $P<0.05$ & $P<0.0001$ & $P<0.05$ \\
\hline $\begin{array}{l}\text { Implantation/ } \\
\text { fetal number }\end{array}$ & $8.25 \pm 1.63$ & $7.71 \pm 1.70$ & $8.00 \pm 1.83$ & $8.43 \pm 0.10^{\mathrm{a}}$ & $6.43 \pm 1.23^{b}$ & $5.14 \pm 0.24^{b}$ & $P<0.05$ & NS & NS \\
\hline
\end{tabular}

Values are presented as mean \pm S.D., $n=8$ dams at each stage of pregnancy. Data were analyzed using two-way ANOVA to determine the main effects and the interactions of diet and time. Pairwise comparison using Bonferroni correction was used to determine differences when there was an observed interaction.

Letters $(\mathrm{a}, \mathrm{b}$ and $\mathrm{c})$ represent significant difference between stages of pregnancy in each dietary group. $P<0.05$ was considered significant.

N/A, not available; NS, not significant.

The authors state that these revisions do not affect results presentation, data interpretation, discussion or conclusion. 